\title{
IRA Y PERSONALIDAD RESISTENTE EN DEPORTISTAS DE RAQUETA Y RESISTENCIA
}

\section{ANGER AND HARDY PERSONALITY IN RACKET AND ENDURANCE SPORTSMAN}

\author{
González-García, H. ${ }^{1}$, Pelegrín, A. ${ }^{2}$ y Carballo, $\mathrm{J}^{3}$. \\ ${ }^{1}$ González-García, H. Doctor por la Universidad Miguel Hernández de Elche. Entrenador de \\ Tenis de mesa en Club Tenis de mesa Ilicitano. higinio.gonzalez@goumh.es \\ ${ }^{2}$ Pelegrín, A. Doctora en Psicología y Profesora en el Departamento de Psicología de la Salud \\ de la Universidad Miguel Hernández. apelegrin@umh.es \\ ${ }^{3}$ Carballo, J. Doctor en Psicología y Profesor en el Departamento de Psicología de la Salud de \\ la Universidad Miguel Hernández. jcarballo@umh.es
}

Código UNESCO: 6199 Otras especialidades psicológicas (Psicología del deporte)

Clasificación Consejo de Europa: 15. Psicología del Deporte

Recibido el 19 de marzo de 2017

Aceptado el 8 de junio de 2017

Correspondencia:

González-García, H.

higinio.gonzalez@goumh.es

\section{RESUMEN}

Los objetivos del presente trabajo de investigación fueron, conocer cuáles son las diferencias en los niveles de personalidad resistente en función del tipo de deporte (raqueta o resistencia) y comprobar cuáles son las diferencias en los niveles de ira en función del tipo de deporte (raqueta o resistencia). La muestra total se compuso de 121 deportistas, de los cuales 58 practican deportes de resistencia y 63 deportes de raqueta. Se utilizó un cuestionario sociodemográfico ad hoc, la Escala de Personalidad Resistente en Maratonianos (EPRM), el Inventario de Expresión de la Ira Estado-Rasgo STAXI-2 y la escala de Oviedo de Infrecuencia de Respuesta (INF-OV). Los resultados mostraron diferencias significativas en el control y compromiso en favor de los deportistas de resistencia. A su vez, el control se relacionó con el deporte de resistencia. Por otro lado, los deportistas de raqueta presentaron más expresión interna de la ira y la expresión interna de la ira se relacionó con la práctica de deporte de raqueta. Se concluyó que los deportistas de resistencia presentan mayores niveles de control y compromiso que los deportistas de raqueta. Además, los deportistas de resistencia obtuvieron menores niveles de expresión interna de la ira que los deportistas de raqueta.

PALABRAS CLAVE: emociones, personalidad, deporte, carácter; ira 


\section{ABSTRACT}

The objectives of this research were to know what are the differences in hardiness levels depending on sport type (racket or endurance) and find out what are the differences in anger levels depending on sport type (racket or endurance). The total sample consisted of 121 athletes, 58 of whom practice endurance sports and 63 racket sports. In this research were used a sociodemographic questionnaire ad hoc, the Marathoners Hardy Personality Scale (EPRM), the Anger State-Trait Expression Inventory (STAXI-2) and the Oviedo Scale of Infrequency Response (INF-OV). The results showed significant differences in control and commitment to endurance athletes. Furthermore, control was associated with endurance sports. On the other hand, racket athletes had more internal anger expression and internal anger expression was associated with racket sport practice. It was concluded that, endurance athletes had higher levels of control and commitment than racket athletes. In addition, endurance athletes had lower levels of internal anger expression than racket athletes.

KEYWORDS: Emotions, personality, sport, character, anger.

\section{INTRODUCCIÓN}

La personalidad resistente es una variable psicológica multidimensional que protege a quien la posee de los efectos del estrés, transformando las experiencias personales en situaciones de desarrollo y crecimiento personal ${ }^{1}$. En el terreno deportivo presenta una gran importancia, ya que el deportista es una persona que debe comprometerse con la tarea diaria del entrenamiento; las situaciones que se producen en el contexto deportivo obligan al deportista a tomar decisiones; y las situaciones adversas que se producen en el deporte, constituyen oportunidades de crecimiento personal al enfrentarse a ellas ${ }^{2}$.

En el ámbito del deporte, los estudios que han examinado a la personalidad resistente con el rendimiento deportivo han mostrado una relación positiva entre ambas variables ${ }^{3-6}$. De esta forma, Golby y Sheard ${ }^{3}$, comprobaron que los deportistas que participaban en ligas superiores tuvieron mayores niveles de Control, Compromiso y Desafío que el resto. Resultados similares encontraron Jaenes et al. $^{4}$, que mostraron que los maratonianos con mejores marcas tenían mejores niveles de personalidad resistente. Al contrario que en los trabajos anteriores, De la Vega et al. ${ }^{7}$ no encontraron diferencias en el rendimiento deportivo y los niveles de personalidad resistente en corredores de diez kilómetros y skyrunning (corredores de alta montaña).

Por otro lado, la ira es otra variable que influye en el rendimiento deportivo de forma positiva y negativa ${ }^{8-10}$. La ira se trata de una emoción que activa al organismo, facilita y potencia el ataque, aunque no provoca siempre agresión ${ }^{11}$. En el caso de los deportes de contacto, el rugby y las tareas de fuerza, la ira se convierte en un facilitador del rendimiento deportivo ${ }^{8,12-14}$. Por otro lado, en los deportes con un alto dominio técnico la ira puede entenderse como facilitador y como depresor del rendimiento deportivo, puede ser entendida como facilitador en el caso de que los deportistas sientan que controlan este excedente de energía $^{15-16}$.

22 IRA Y PERSONALIDAD RESISTENTE EN DEPORTISTAS DE RAQUETA Y RESISTENCIA 
Al mismo tiempo, la ira también influye en la elección deportiva y los niveles de ira son diferentes en los deportistas individuales y de equipo ${ }^{17,18}$. De manera que, hay diversos trabajos que han mostrado mayores niveles de ira en los deportes colectivos y menores en los individuales ${ }^{18}$. Por otra parte, Maxwell et al. ${ }^{17}$ encontraron que los deportistas con mayores niveles de ira, fueron tendentes a elegir más el rugby que los deportes de contacto e individuales.

Por todo lo expuesto anteriormente, la ira y la personalidad resistente se muestran como dos variables de interés en el análisis del rendimiento en el deporte de raqueta y resistencia. Por ello, es necesario conocer los diferentes perfiles de deportistas en estas dos modalidades deportivas. Por lo tanto, los objetivos que se pretenden alcanzar son: conocer cuáles son las diferencias en los niveles de personalidad resistente en función del tipo de deporte (raqueta o resistencia), y comprobar cuáles son las diferencias en los niveles de ira en función del tipo de deporte (raqueta o resistencia).

\section{MÉTODO}

\section{Participantes}

La muestra total se compuso de 121 deportistas, aunque se eliminaron 25 participantes que habían contestado de forma deshonesta al cuestionario. De total, 58 practican deportes de resistencia (Ciclismo y Triatlón) y 63 deportes de raqueta (tenis, frontón, pádel y tenis de mesa), 15 son mujeres $(12,4 \%$ ) y 106 son hombres $(87,6 \%)$ con una edad comprendida entre los 18 años y los 64 años $(M=31,52 ; \mathrm{DT}=10,98)$. De ellos, 46 eran no federados $(38 \%), 75$ eran federados $(62 \%)$ y 15 eran deportistas profesionales $(12,4 \%)$.

\section{Variables e Instrumentos}

Tipo de Deporte. Para evaluar los factores sociodemográficos y el tipo de deporte, se creó un cuestionario sociodemográfico ad hoc. Los ítems valoraron aspectos relacionados con: variables biológicas del deportista (altura, sexo, edad, etc.); variables laborales y académicas (nivel de estudios, situación laboral, etc.) y variables sociodeportivas (deporte, éxitos deportivos, profesional o amateur, etc).

Aquiescencia y participantes deshonestos. La escala de Oviedo de infrecuencia de respuesta (INF-OV $)^{19}$, es una medida de autoinforme que está compuesta por 12 ítems que consta de una escala likert con 5 posibilidades ( $1=$ "Completamente en desacuerdo"; 5 = "Completamente de acuerdo"). Los participantes con más de 3 respuestas incorrectas fueron retirados de la muestra.

Evaluación de la Ira. Para la medición de la Ira Rasgo, Expresión Externa de Ira, Expresión Interna de Ira, Temperamento, Reacción de Ira, Control Interno de Ira, Control Externo de Ira y del Índice de Expresión de la Ira, se utilizó el 
Inventario de Expresión de la Ira Estado-Rasgo STAXI-2 ${ }^{20}$. En concreto, la escala de Ira Rasgo obtuvo una correlación test-retest a los dos meses de ,71 y un coeficiente alfa de Cronbach de ,82 lo que indica niveles apropiados de fiabilidad de estabilidad temporal y de consistencia interna, respectivamente.

Personalidad Resistente. Para su medición se empleó la escala de Personalidad Resistente en Maratonianos (EPRM) ${ }^{21}$. La Escala de Personalidad Resistente en Maratonianos (EPRM) tiene formato tipo Likert con 4 alternativas de respuesta, desde $0=$ "totalmente en desacuerdo", a $3=$ "totalmente de acuerdo". El instrumento resulta válido y fiable, con una consistencia interna para la Escala Completa de ,79; de ,71 para Compromiso; de ,59 para Control y de ,42 para Desafío.

\section{Procedimiento}

En primer lugar, el estudio fue aprobado por el comité ético de la Universidad Miguel Hernández de Elche. Posteriormente, se contactó con las federaciones deportivas españolas, a nivel online; y con entrenadores y deportistas, a nivel presencial. Las federaciones anunciaron en su web las condiciones para participar en el estudio. De esta forma los deportistas interesados contactaban con el investigador principal. Los participantes voluntarios, enviaban un email de participación a los investigadores, y una vez aseguraban su interés en participar, recibían el enlace al cuestionario de investigación. Por otro lado, en el caso de contactar con los deportistas y entrenadores a nivel presencial, los deportistas daban su email a los investigadores para recibir las instrucciones y el cuestionario de investigación en su correo electrónico. En ambos casos, el cuestionario podían realizarlo libremente a través de internet en su tiempo libre. Una vez los participantes accedían al cuestionario, firmaban un consentimiento informado. Posteriormente a firmar el consentimiento informado, comenzaban a completar el cuestionario de investigación. Una vez finalizado el cuestionario, los datos se alojaban a través de la aplicación «Google Drive». En la que se guardaron los cuestionarios de investigación en formato electrónico de Excel.

\section{Análisis de Datos}

El análisis de datos se llevó a cabo mediante el programa SPSS 19,0. Se realizaron los estadísticos descriptivos de media, mínimo, máximo, frecuencias, porcentaje y desviación típica, para conocer las características de la muestra. La prueba $t$ de Student para muestras independientes, se utilizó para conocer las diferencias de medias cuando las variables eran cuantitativas, empleándose un nivel de confianza del 95\%. La regresión lineal, se empleó con la finalidad de estimar el valor predictivo de las variables de ira y de personalidad resistente sobre el tipo de deporte (raqueta o resistencia). La $d$ de Cohen, se utilizó para analizar el tamaño del efecto y así conocer la magnitud de las diferencias encontradas en la prueba $t$ de Student. Siguiendo a Cohen ${ }^{22}$ podemos considerar los resultados del tamaño del efecto de la siguiente forma: $d=, 20$ (pequeño), $d=, 50$ (moderado) y $d=, 80$ (grande). 


\section{RESULTADOS}

En primer lugar, con el objetivo de conocer cuáles son las diferencias en los niveles de personalidad resistente en función del tipo de deporte (raqueta o resistencia), se realizó una prueba $t$ para muestras independientes, en la que subdividió a la muestra en deportes de raqueta (DR; tenis, frontenis, pádel y tenis de mesa) y deportes de resistencia (DC; ciclismo y triatlón).

Tabla 1. Personalidad Resistente y Tipo de Deporte

\begin{tabular}{ccccc}
\hline Variables de & $\mathrm{DC}$ & $\mathrm{DR}$ & & $\mathrm{d}$ \\
Personalidad & $(\mathrm{N}=58)$ & $(\mathrm{N}=63)$ & $\mathrm{t}(\mathrm{p})$ & Cohen \\
Resistente & $\mathrm{M}(\mathrm{DT})$ & $\mathrm{M}(\mathrm{DT})$ & & \\
\hline
\end{tabular}

$\begin{array}{llll}\text { Personalidad } & 64,10(8,57) & 61,26(8,78) & 1,79(, 075)\end{array}$

Control

$22,98(2,74) \quad 21,93(3,01) \quad 1,99(, 049)^{*} \quad, 36$

Compromiso

$23,51(3,32)$

$21,65(4,30)$

$2,68(, 008)^{* *}$ , 49

Desafío

$17,60(3,99)$

$17,68(3,95)$

$-, 109(, 913)$

Nota. DC = Deportes Cíclicos; DR = Deportes de Raqueta . $p<, 05 ;{ }^{* *} p<, 01$

En la Tabla 1 , el control $(p<, 05)$ y compromiso $(p<, 01)$ reportaron diferencias significativas y un tamaño del efecto pequeño, en favor de los deportistas de resistencia que obtuvieron mayores niveles.

En la Tabla 2, para conocer el valor predictivo de cada una de las variables estadísticamente significativas en la prueba $t$, que en este caso fueron el compromiso y el control, se realizó una regresión lineal. El modelo predictivo ha resultado significativo $(F=413,18 ; p<, 01)$. Además, la capacidad predictiva del modelo resultó muy elevada $\left(R^{2}=, 87\right)$, siendo en este caso la variable control la que influye en la práctica del deporte de resistencia. 
Tabla 2. Regresión lineal para predecir el valor del compromiso y el control, sobre el tipo de deporte.

$\begin{array}{llllll}\text { Variables } & \text { B } & \text { Error típ. } & \text { Beta } t & P\end{array}$

\begin{tabular}{cccccc}
\hline Compromiso &,- 000 &, 015 &,- 028 &,- 133 &, 895 \\
Control &, 068 &, 015 &, 963 & $4,558,000^{* *}$
\end{tabular}

Nota. ${ }^{*} \mathrm{p}<, 05 ;{ }^{* *} \mathrm{p}<, 01$

En segundo lugar, con el objetivo de comprobar cuáles son las diferencias en los niveles de ira en función del tipo de deporte (raqueta o resistencia), se realizó una prueba $t$ para muestras independientes, en la que subdividió a la muestra en deportes de raqueta (DR; tenis, frontenis, pádel y tenis de mesa) y deportes de resistencia (DC; ciclismo y triatlón).

Tabla 3. Variables de Ira y Tipo de Deporte

\begin{tabular}{|c|c|c|c|c|}
\hline Variables de Ira & $\begin{array}{c}\mathrm{DC} \\
(\mathrm{N}=58) \\
\mathrm{M}(\mathrm{DT})\end{array}$ & $\begin{array}{c}\text { DR } \\
(\mathrm{N}=63) \\
\mathrm{M}(\mathrm{DT})\end{array}$ & $t(p)$ & $\begin{array}{c}d \\
\text { Cohen }\end{array}$ \\
\hline Ira Rasgo & $21(5,16)$ & $22,14(4,87)$ & $\begin{array}{c}-1,248 \\
(, 214)\end{array}$ & \\
\hline Temperamento & $8,08(2,96)$ & $8,61(3,11)$ &,$- 962(, 338)$ & \\
\hline Reacción de Ira & $12,91(3,42)$ & $13,52(3,02)$ & $-1,041(, 30)$ & \\
\hline Expresión Externa Ira & $10,72(2,85)$ & $11,66(2,89)$ & $\begin{array}{r}-1,803 \\
(, 074)\end{array}$ & \\
\hline Expresión Interna Ira & $12,81(3,54)$ & $14,36(3,18)$ & $\begin{array}{l}-2,543 \\
(, 012)^{*}\end{array}$ & ,46 \\
\hline Control Externo Ira & $19,89(3,23)$ & $19,47(3,33)$ & ,703 (,483) & \\
\hline Control Interno Ira & $16,55(3,71)$ & $16,42(3,70)$ & ,182 (,856) & \\
\hline Índice Expresión de Ira & $23,08(8,48)$ & $26,12(9,41)$ & $-1,86(, 065)$ & \\
\hline
\end{tabular}

Nota. DC = Deportes Cíclicos; DR = Deportes de Raqueta. $\mathrm{p}<, 05 ;{ }^{* *} \mathrm{p}<, 01$

En la Tabla 3, la expresión interna de la ira $(p<, 05 ; d=, 46)$ reportó diferencias significativas y un tamaño del efecto pequeño, en favor de los deportistas de resistencia.

En la Tabla 4, para conocer el valor predictivo de la expresión interna de la ira sobre el tipo de deporte, se realizó una regresión lineal. En relación a los resultados obtenidos, el modelo predictivo ha resultado significativo ( $F=889,38$; $\mathrm{p}<, 01)$. Además, la capacidad predictiva del modelo resultó muy elevada $\left(\mathrm{R}^{2}=\right.$ ,881), siendo en este caso la variable expresión interna de la ira la que predice la práctica de deporte de resistencia. 
Tabla 4. Regresión lineal para predecir el valor de la Expresión Interna de la Ira, sobre el tipo de deporte.

\begin{tabular}{cccccc}
\hline Variables & B & $\begin{array}{c}\text { Error Beta } \\
\text { típ. }\end{array}$ & $t$ & $p$ \\
\hline Expresión Interna de la Ira &, 107 &, 004 &, 939 & 29,82 &, $000^{* *}$ \\
\hline
\end{tabular}

Nota. $\mathrm{p}<, 05 ; \mathrm{p}<, 01$

\section{DISCUSIÓN}

El primer objetivo de este trabajo de investigación fue, conocer cuáles son las diferencias en los niveles de personalidad resistente en función del tipo de deporte (raqueta o resistencia). Los deportistas de resistencia obtuvieron mayores niveles de control y compromiso, pero no obtuvieron diferencias significativas en los factores desafío y personalidad resistente. Por lo tanto, se puede afirmar que los deportistas de resistencia se sienten más comprometidos con su entrenamiento y sienten mayor control de su entrenamiento diario, que los deportistas de raqueta. Estos resultados se entienden desde la perspectiva de que, la mayoría de los deportistas de resistencia forman parte activa en la planificación y programación de su preparación. Es decir, son mucho más autónomos en su entrenamiento que los deportistas de raqueta, lo que puede influir en tener mayores niveles de compromiso y control. Además, la literatura anterior ya había mostrado mayores niveles de personalidad resistente, en deportistas de resistencia que en la población general ${ }^{4,7}$. Por lo tanto, también se muestra que los deportistas de resistencia presentan mayor compromiso y control que los de raqueta.

El segundo objetivo de investigación fue, comprobar cuáles son las diferencias en los niveles de ira en función del tipo de deporte (raqueta o resistencia). En este sentido, los deportistas de raqueta obtuvieron mayores niveles de expresión interna de la ira. En el caso de los deportes de raqueta, durante el juego se dan numerosas situaciones que favorecen el aumento de los niveles de ira por el deportista. En consecuencia, estas situaciones potencian las posibilidades de expresar la ira de manera interna. Al contrario, en los deportes de resistencia el entorno es mucho más estable, existiendo menos situaciones que potencien la expresión de la ira en cualquiera de sus dos vertientes: externa o interna. Por otra parte, la literatura anterior encontró diferencias según el tipo de deporte en los niveles de ira y encontró relación entre los niveles de ira y la elección deportiva ${ }^{13,17}$, pero no se encuentran estudios que analicen las diferencias en los niveles de ira entre los deportes de raqueta y resistencia. Por lo tanto, en el presente trabajo de investigación se confirma que los deportistas de raqueta poseen mayor expresión interna de la ira que los deportistas de resistencia. son:

Las conclusiones que se sustraen del presente trabajo de investigación 
- Los deportistas de resistencia presentan mayores niveles de control y compromiso que los deportistas de raqueta.

- Los deportistas de resistencia obtuvieron menores niveles de expresión interna de la ira que los deportistas de raqueta.

Como líneas de propuesta futuras se presentan las siguientes:

- Replicar el estudio ampliando el número de deportes de raqueta y de deportes de resistencia de la muestra. De esta forma se podrían crear perfiles por deportes.

- Repetir el estudio, pero centrándose en deportes concretos de resistencia y raqueta, para conocer las diferencias que existen entre los diferentes deportes de raqueta y resistencia entre sí.

Entre las limitaciones principales del presente trabajo destaca, la imposibilidad de captar un número significativo de participantes de todas las disciplinas de raqueta y resistencia, por el gran número de modalidades existentes. Por otro lado, la realización del estudio online puede disminuir la aquiescencia de las respuestas obtenidas, pero para ello se utilizó la escala de Oviedo de Infrecuencia de Respuesta (INF-OV) para garantizar la honestidad de las respuestas obtenidas.

\section{REFERENCIAS BILIOGRÁFICAS}

1. Eschleman KJ, Bowling NA, Alarcon GM. A meta-analytic examination of hardiness. International Journal of Stress Management. 2010;17(4):277-307.

2. Jaenes JC. Personalidad resistente en deportes. Revista Andaluza de Medicina del Deporte. 2009;2(3):98-101.

3. Golby J, Sheard M. Mental toughness, and hardiness at different levels of rugby league. Personality and Individual Differences. 2004;37:933-42.

4. Jaenes JC, Godoy-Izquierdo D, Román, FM. Personalidad Resistente en maratonianos: un estudio sobre el control, compromiso y desafío en corredores y corredoras de maratón. Revista de Psicología del Deporte. 2009;18(2):217-34.

5. Rezae A, Ghaffari M, Zolfalifam J. Research Journal of Biological Sciences. 2009;4(9):10105.

6. Sheard M, Golby J. Personality hardiness differentiates elite-level sport performance. International Journal of Sport and Exercise Psychology. 2010;8:160-9.

7. De la Vega R, Rivera O, Ruiz R. Personalidad Resistente en Carreras de Fondo: comparativa entre ultra fondo y diez kilómetros. Revista de Psicología del Deporte. 2010;20(2):445-54.

8. Davis PA, Woodman T, Callow N. Better out than in: the influence of anger regulation on physical performance. Personality and Individual Differences. 2010;49(5):57-460. doi: 10.1016/j.paid.2010.04.017

9. Parrott W. Emotions in Social Psychology. Philadelphia: Psychology Press; 2001.

10. Tamir M, Mitchell C, Gross JJ. Hedonic and Instrumental Motives in Anger Regulation. Psychological Science. 2008;19:324-8.

11. Berkowitz L. Agresión: causas, consecuencias y control. Bilbao: Desclée de Brouwer; 1996.

12. Oliva F, Calleja N, Hernández-Pozo, R. Escala de creencias sobre la ira en el deporte de combate con atletas mexicanos. Revista Internacional de Medicina y Ciencias de la Actividad Física y el Deporte. 2012;12(45):110-21.

13. Robazza C, Bortoli L. Perceived impact of anger and anxiety on sporting performance in rugby players. Psychology of Sport and Exercise. 2007;8:875-96.

14. Ruiz MC, Yuri HL. Perceived impact of anger on performance of skilled karate athletes. Psychology of Sport and Exercise. 2011;12(3):242-9. doi:10.1016/j.psychsport.2011.01.005. 
15. Hanton S, Jones G, Mullen R. Intensity and direction of competitive state anxiety as interpreted by rugby players and rifle shooters. Perceptual and Motor Skills. 2000; 90:51321.

16. Martinent G, Ferrand C. A naturalistic qualitative study of the directional interpretation process of discrete emotions during high-stakes table tennis matches. Journal of Sport and Exercise Psychology. 2009;31:318-36.

17. Maxwell JP, Visek, AJ, Moores E. Anger and perceived legitimacy of aggression in male Hong Kong Chinese athletes: Effects of type of sport and level of competition. Psychology Sport and Exercise. 2009;10:289-96.

18. Pelegrín A, Serpa S, Rosado A. Aggressive and unsportsmanlike behaviours in competitive sports: an analysis of related personal and environmental variables. Anales de Psicología. 2013;29(3):701-13. doi: http://dx.doi.org/10.6018/analesps.29.3.175841.

19. Fonseca-Pedrero E, Lemos-Giráldez S, Paino M, Villazón-García U, Muñiz J. Validation of the Schizotypal Personality Questionnarie Brief form in adolescents. Schizophrenia Research. 2009;111(1-3):53-60.

20. Spielberger CD, Miguel-Tobal JJ, Casado MI, Cano-Vindel A. Inventario de Expresión de Ira Estado Rasgo 2-STAXI 2. Madrid: TEA; 2001.

21. Jaenes JC, Godoy-Izquierdo D, Román FJ. Elaboración y validación psicométrica de la escala de Personalidad Resistente en Maratonianos (EPRM). Cuadernos de Psicología del Deporte. 2008;8(2):59-81.

22. Cohen J. Statistical Power Analysis for the Behavioral Sciences. New York: Academic Press; 1988.

Referencias totales citadas: $\mathbf{2 2}$

Referencias citadas correspondientes a la Rev lb CC Act Fis Dep: 0 